\title{
Optimization and Evaluation of Monodispersed Tetrandrine- Loaded PLA Microspheres Prepared With A SPG Membrane Emulsification Technique
}

\author{
Jin Lu ${ }^{1,2,3}$, Ai-ling Yin ${ }^{1,2}$, Meng Zhang ${ }^{1,2}$, Xue-e Yu' ${ }^{1,2}$, Ting-ming Fu ${ }^{1,2}$, Hua-xu Zhu ${ }^{1,2}$ and Li-wei \\ $\mathrm{Guo}^{1,2 *}$ \\ ${ }^{1}$ Jiangsu Collaborative Innovation Center of Chinese Medicinal Resources Industrialization, Nanjing University of Chinese Medicine, Nanjing 210023, \\ China \\ ${ }_{2}^{2}$ Jiangsu Botanical Medicine Refinement Engineering Research Center, Nanjing University of Chinese Medicine, Nanjing 210023, China \\ ${ }^{3}$ Zhongda Hospital Southeast University, Nanjing 210000, China
}

Received: October 10, 2016; Accepted: November 11, 2016; Published: January 02, 2017

*Corresponding author: Li-wei Guo, Nanjing University of Chinese Medicine, Nanjing 210023, China, Tel: +86 25 86798188;

E-mail: guoliwei815@126.com

\begin{abstract}
To improve pulmonary drug concentrations and to maximize the effectiveness and minimize the adverse side effects, uniformsized tetrandrine-loaded polylactide (PLA) microspheres with a suitable particle size for pulmonary inhalation were prepared by the Shirasu Porous Glass (SPG) membrane emulsification technique. The main parameters influencing PLA microsphere properties were investigated: transmembrane pressure, circulation speed, high hydrophilic-lipophilic balance (HLB value), PLA concentration and oil-water volume ratio. Narrowly size-distributed tetrandrine-loaded PLA microspheres were obtained with a high drug encapsulation efficiency of $81.0 \%$ and an average diameter of $3.16 \mu \mathrm{m}$ under optimized conditions. The results of Fourier transform infrared, differential scanning calorimetry and powder X-ray diffraction revealed that tetrandrine would be either molecularly dispersed in the polymer or distributed in an amorphous form. Further, the in vitro drug release experiment confirmed that the uniform-sized tetrandrine-loaded PLA microspheres showed suppressed burst release. These studies provide a basis for the use of uniformly sized $3.16 \mu \mathrm{m}$ tetrandrine-loaded PLA microspheres for pulmonary inhalation.
\end{abstract}

Keywords: Tetrandrine; Membrane emulsification; PLA microspheres; Encapsulation efficiency; Characterization; Pulmonary inhalation

\section{Introduction}

Tetrandrine, with the molecular formula of $\mathrm{C}_{33} \mathrm{H}_{42} \mathrm{~N}_{2} \mathrm{O}_{6}$ (Fig. 1), a bisbenzylisoquinoline alkaloid isolated from the root of Fangji (Stephania tetrandra S. Moore) [1], has been broadly used in China to treat patients with arthritis, hypertension and inflammation [2-3]. Previous studies have reported that tetrandrine has antitumor activity, both in cultured tumor cells and in animal models and has potential as a cancer chemotherapeutic agent, especially in lung cancer treatment [45]. However, tetrandrine belongs to the BCS class II category of drugs, which have low aqueous solubility, resulting in low oral bioavailability. An ordinary tetrandrine injection distributes widely in vivo without a targeting effect, thus tetrandrine in this formulation does not concentrate highly in diseased organs or tissues [6-7] and usually causes pain or phlebitis. To improve pulmonary drug concentration to maximize its effectiveness and minimize the adverse side effects, tetrandrine polylactic acid microspheres with diameters of 7-15 $\mu \mathrm{m}$ were injected intravenously to achieve lung targeting [8]. This mechanism of lung targeting by injecting more than $7 \mu \mathrm{m}$ microspheres occurs through mechanical filtration by the pulmonary capillaries, which can damage the lung permanently. Microspheres with diameters between 0.5-5 $\mu \mathrm{m}$ used for pulmonary inhalation could overcome this shortcoming. However, the controllability and uniformity of the microsphere size are closely related to the effects of pulmonary inhalation. The particle size is difficult to control for microspheres prepared by conventional methods, such as emulsification, ultrasonic emulsifying preparation and distribution [9].

Recently, a novel method, the Shirasu Porous Glass (SPG) membrane emulsification technique, has been widely applied to the preparation of uniform-sized emulsions. Uniform droplet size and high drug encapsulation efficiency have been obtained by permeating a dispersed phase into a continuous phase through an SPG membrane at an adequate pressure [10-14].

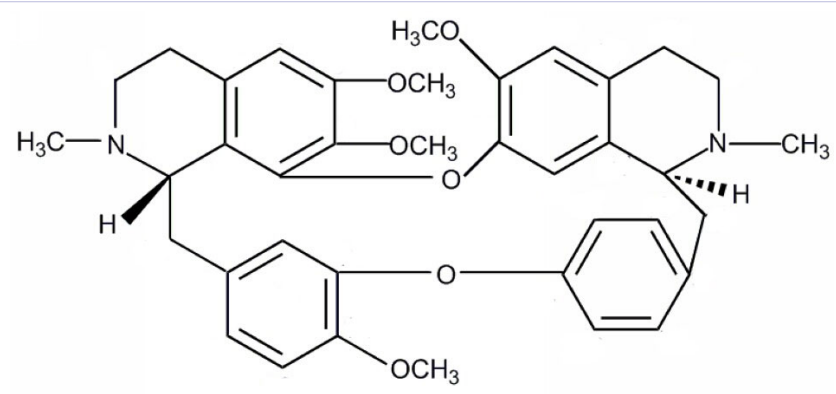

Figure 1: The chemical structure of tetrandrine. 
Furthermore, the microsphere size can be controlled within 2-10 $\mu \mathrm{m}$ and optimized to within 2-5 $\mu \mathrm{m}$; this size is most effective for inhalation applications, as particles over $5 \mu \mathrm{m}$ are limited to the upper airways by inertial impaction [15-16]. This novel method is advantageous for several important reasons: (1) controlled droplet size and narrow droplet size distribution are possible under the proper conditions [17-18], (2) the practical and theoretical evaluation of properties, such as the drug release rate, would become simple and precise with uniformsized microcapsules, and the burst effect could be avoided [1920], and (3) owing to its ease of operation, simple equipment, mildness without violent stirring and low energy consumption during the process, many drugs sensitive to light, heat, oxygen or collision have been applied in this method [21-23]. However, this novel method has not been fully applied to the active ingredients of traditional Chinese medicine, which contribute significantly to pharmaceutical industries. Although paclitaxel and hydroxycamptothecin have been applied with this method, the drug encapsulation efficiency was below $80 \%$ [24].

The objective of this study was to develop uniform-sized tetrandrine-loaded polylactide (PLA) microspheres prepared by the Shirasu Porous Glass (SPG) membrane emulsification technique with the emulsion-solvent evaporation method for pulmonary inhalation. The main parameters (the transmembrane pressure, the circulation speed, the HLB value, the PLA concentration and the oil-water volume ratio) were investigated by a single factor test and orthogonal experiments to obtain uniform-sized and size controlled tetrandrine-loaded PLA microspheres with a high encapsulation efficiency. Furthermore, the reproducibility and stability of the microspheres prepared under optimized conditions were investigated. Fourier transform infrared, differential scanning calorimetry and powder X-ray diffraction analysis were used to evaluate the properties of the microspheres. Finally, the in vitro experiment revealed the drug release behavior of the tetrandrine-loaded PLA microspheres.

\section{Materials and methods}

\section{Materials}

The tetrandrine reference substance (Batch 110711-200708) was purchased from the National Institute for the Control of Pharmaceutical and Biological Products (NICPBP, Beijing, China). The tetrandrine (purity $>98.0 \%$ ) raw material was received from Nanjing Zelang Medical Technology Co., Ltd. (Nanjing, China). Polylactide (PLA), with an average molecular weight $(\mathrm{Mw})$ of $10 \mathrm{kDa}$, was purchased from Shandong Institute of Medical Instruments (Shandong, China). Polyvinyl alcohol (PVA, degree of polymerization 1700 , degree of hydrolysis $87.0 \%$ 89.0\%) was provided by Aladdin Reagent Co., Ltd. (Shanghai, China). Dichloromethane (DCM) was ordered from Nanjing Chemical Reagent Co., Ltd (Nanjing, China). Gelatin was ordered through chemical reagent procurement and the supply station packing plant (Shanghai, China). Tween-20 and Tween-80 were purchased from Tianjin Kermel Chemical Reagent Co., Ltd (Tianjin, China). Sodium dodecyl sulfate (SDS) was supplied by Shanghai Lingfeng Chemical Reagent Co., Ltd. (Shanghai, China).
All of the other materials were of analytical reagent grade and used without further purification.

\section{Methods}

\section{Preparation of PLA microspheres}

Experimental set-up and procedure: A miniature kit for SPG emulsification was made in the laboratory, as illustrated in Fig. 2, a tube-shaped hydrophilic SPG membrane, with an outer diameter of $10 \mathrm{~mm}$, a thickness of $1.0 \mathrm{~mm}$ and a pore size of $1.9 \mu \mathrm{m}$, was purchased from SPG technology (Miyazaki, Japan). PLA and the drug (tetrandrine-free PLA microspheres without the drug) were resolved in dichloromethane as the dispersed phase (oil phase), was stored in a pressure-tight vessel, connected to a nitrogen gas inlet attached to a pressure gauge, and extruded through the uniform pores of the membrane at an adequate pressure. The continuous phase (water phase), containing $0.5 \%$ surfactant (one type of gelatin, SDS, Tween-20 or Tween-80) and $0.5 \%$ PVA, was circulated inside the SPG membrane with a peristaltic pump. Then, as shown in Fig. 3, the droplets were detached from the membrane surface, dispersed in the immiscible continuous phase, stabilized by the PVA and surfactant inside, and then solidified by simply evaporating the solvent at room temperature. To remove the untrapped drug and PVA, the suspension was washed with deionized water followed

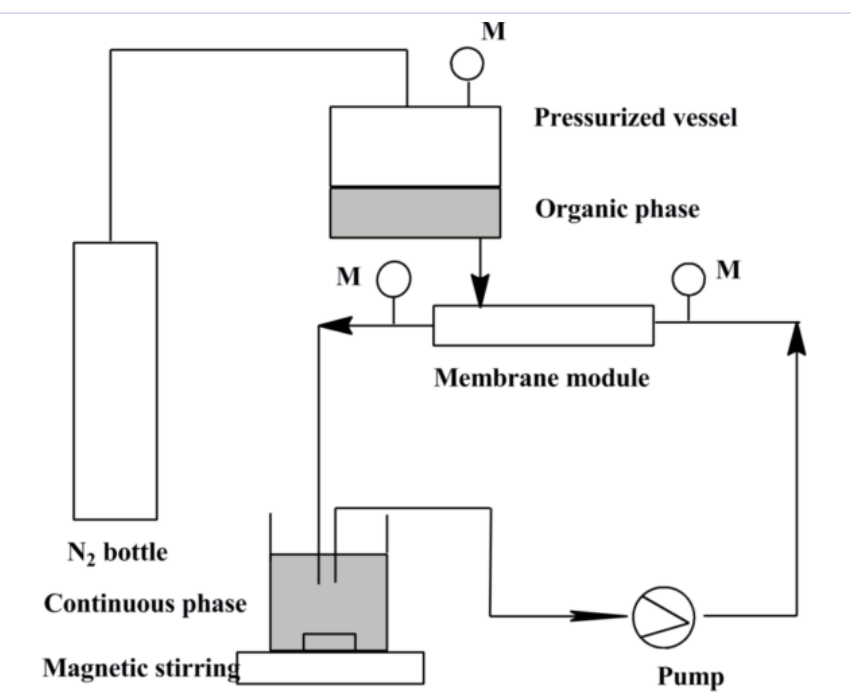

Figure 2: Schematic diagram of a miniature kit for SPG membrane emulsification.

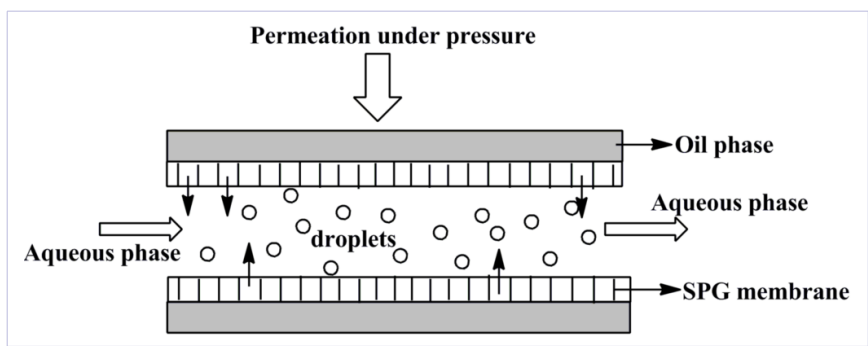

Figure 3: Schematic diagram of the principle. 
by centrifugation at $15000 \mathrm{rpm}$ for $45 \mathrm{~min}$. This procedure was repeated three times. The resulting PLA microspheres with little deionized water were freeze-dried over $48 \mathrm{~h}$ under a freedrier system to obtain PLA microspheres in a powder form. Single factor experimental design

The experiments were carried out over a transmembrane pressure between 20-140 MPa, a mild circulation speed (500$2000 \mathrm{rpm}$ ), an HLB value between 9-40, PLA concentration ranging from 5-40 g/L and an oil-water volume ratio of 1:5-1:40. The specific values for each factor in the single factor experiments are shown in Table 1.

\section{Orthogonal experimental design}

On the basis of the investigations above, orthogonal experiments were designed as shown in table 2; four parameters that played significant roles in the microspheres' properties were chosen: the PLA concentrations, the concentration ratio of tetrandrine to PLA, the SDS concentration and the oil-water volume ratio.

The reproducibility experiment of tetrandrine-loaded PLA microspheres

According to the results from the orthogonal experiments, tetrandrine-loaded PLA microspheres were prepared three times at the optimized conditions and then described with respect to their average size, span value and encapsulation efficiency.

Table 1: The specific values for each factor in single factor experiments.

\begin{tabular}{|c|c|c|c|c|}
\hline $\begin{array}{l}\text { Transmembrane } \\
\text { pressure } \\
(20-140 \mathrm{MPa})\end{array}$ & $\begin{array}{l}\text { Circulation } \\
\text { Speed } \\
(500-2000 \\
\text { rpm) }\end{array}$ & $\begin{array}{l}\text { HLB value } \\
(9-40)\end{array}$ & $\begin{array}{l}\text { PLA } \\
\text { Concentration } \\
(5-40 \mathrm{~g} / \mathrm{L})\end{array}$ & $\begin{array}{l}\text { Volume } \\
\text { ratio of } \\
\text { oil-water } \\
(v / v)\end{array}$ \\
\hline 0.02 & 500 & $\begin{array}{l}9.8 \\
(0.5 \% \text { gelatin } \\
+0.5 \% \mathrm{PVA})\end{array}$ & 5 & $1: 5$ \\
\hline 0.06 & 1000 & $\begin{array}{l}15 \\
(0.5 \% \\
\text { Tween-80 + } \\
0.5 \% \text { PVA) }\end{array}$ & 10 & $1: 10$ \\
\hline 0.1 & 1500 & $\begin{array}{l}16.7 \\
(0.5 \% \\
\text { Tween-20 + } \\
0.5 \% \text { PVA) }\end{array}$ & 20 & $1: 20$ \\
\hline 0.14 & 2000 & $\begin{array}{l}40 \\
(0.5 \% \text { SDS + } \\
0.5 \% \text { PVA })\end{array}$ & 40 & $1: 40$ \\
\hline
\end{tabular}

Table 2: Designed factors and level values in orthogonal experiments.

\begin{tabular}{|l|l|l|l|l|}
\hline Factors & $\begin{array}{l}\text { Tetrandrine-PLA } \\
\text { (g/g) }\end{array}$ & $\begin{array}{l}\text { SDS } \\
\text { concentration } \\
\text { (g/L) }\end{array}$ & $\begin{array}{l}\text { PLA } \\
\text { concentration } \\
\text { (g/L) }\end{array}$ & $\begin{array}{l}\text { Volume } \\
\text { ratio of } \\
\text { oil-water } \\
\text { (v/v) }\end{array}$ \\
\hline Level 1 & $1: 2$ & 0.3 & 20 & $1: 10$ \\
\hline Level 2 & $1: 4$ & 0.4 & 40 & $1: 30$ \\
\hline Level 3 & $1: 6$ & 0.5 & 60 & $1: 50$ \\
\hline
\end{tabular}

The preliminary stability experiment of tetrandrine-loaded PLA microspheres

The freeze-dried tetrandrine-loaded PLA microspheres were kept at $-20{ }^{\circ} \mathrm{C}, 4{ }^{\circ} \mathrm{C}$ and $37{ }^{\circ} \mathrm{C}$ for 1,2 and 3 months, to evaluate their stability, respectively. The encapsulation efficiency and microscopic appearance of these samples were described.

\section{In vitro characterization of tetrandrine -loaded PLA microspheres}

Morphological examination: The morphological aspects of microspheres were evaluated using scanning electron microscopy (SEM). The freeze-drying microspheres were redispersed in distilled water, then deposited a drop of this water suspension uniformly on a piece of aluminum foil, and they were dried naturally at room temperature, fixed on aluminum foil on the sample stage by conductive glue, and finally observed by scanning electron microscopy (S-4800, Hitachi, Japan).

Determination of average droplet size and droplet size distribution

Microspheres were dispersed in distilled water and analyzed by a laser diffraction particle size analyzer (S3500, Microtrac, America). The microspheres' size and size distribution are expressed as the average diameter and the span factor, respectively. In addition, the span factor was calculated according to Eq. (A.1) [25]. Span values below or equal to 1 are more likely to obtain a narrow size distribution, which is the desired result

$$
\text { Span }=\frac{d_{90}-d_{10}}{d_{50}}
$$

The $d 10, d 50$ and $d 90$ represent the diameters of 10,50 and 90 vol.\% on a relative cumulative microsphere size distribution curve, respectively.

\section{Determination of encapsulation efficiency (EE) and drug loading (DL) percentages}

The precisely weighed freeze-dried tetrandrine-loaded PLA microspheres were dissolved in DCM to scroll 3 min until the microspheres were ruptured completely and were then added in a certain amount of $0.1 \mathrm{M}$ hydrochloric acid solution and scrolled that tetrandrine could be sufficiently dissolved in the hydrochloric acid solution. The supernatant liquid was collected as the sample after centrifugation at $8000 \mathrm{rpm}$ for $5 \mathrm{~min}$. Then, the sample was analyzed by UV spectrophotometer $(\lambda \max =280.0$ $\mathrm{nm})$. The encapsulation efficiency of the tetrandrine-loaded PLA microspheres was calculated according to Eq. (B.1), and the drug loading efficiency was calculated according to Eq. (B.2) [26]. Each experiment was repeated three times, and the results were averaged.

$$
\text { Encapsulation efficiency }=\frac{E_{1}}{E_{2}} * 100 \% \quad \text { (B.1) }
$$

$E_{1}, E_{2}$ represent the practical content of tetrandrine and the theoretical content of tetrandrine, respectively.

$$
\text { Loading efficiency }=\frac{W_{1}}{W_{2}} * 100 \%
$$


$W_{1}$ and $W_{2}$ represent the practical content of tetrandrine and total content of tetrandrine-loaded PLA microspheres, respectively.

\section{Fourier transform infrared analysis}

Fourier transform infrared (FTIR) spectral studies were performed in a Fourier transform infrared spectrometer (AVATA360, Nicolet, America) in the mid-IR range from $500 \mathrm{~cm}^{-1}$ to $4000 \mathrm{~cm}^{-1}$, with a resolution of $4 \mathrm{~cm}^{-1}$.

\section{Differential scanning calorimetry}

Differential scanning calorimetry (DSC) measurements were carried out with a differential scanning calorimeter (DSC204, Bruker, Germany). Samples (10 mg) were separately sealed in a standard aluminum pan and heated in an inert atmosphere of nitrogen with a temperature ramp speed of $10.0^{\circ} \mathrm{C} / \mathrm{min}$. The heat flow was recorded from 30 to $380^{\circ} \mathrm{C}$.

\section{Powder X-ray diffraction (XRD) study}

XRD experiments were carried out to characterize the physical state of tetrandrine in tetrandrine-loaded PLA microspheres using an intelligent X-ray diffractometer (XRD-6000, Shimadzu, Japan). Approximately $5 \mathrm{mg}$ of each of the raw, physical mixtures of tetrandrine and PLA, tetrandrine-free PLA microspheres and tetrandrine-loaded PLA microspheres were studied. XRD studies were performed by exposing samples to CuKa radiation $(40 \mathrm{kV}$, $100 \mathrm{~mA}$ ) and scanned from $5^{\circ}$ to $50^{\circ}, 2 \theta$ at a scan step of $0.02^{\circ}$ and a scan speed of $20^{\circ} / \mathrm{min}$.

\section{In vitro tetrandrine release study}

To evaluate the stability of the microspheres, an accurate amount of tetrandrine-loaded PLA microspheres were redispersed in $5 \mathrm{~mL}$ of saline within a dialysis bag, then disposed in $195 \mathrm{~mL} 0.1 \mathrm{M}$ hydrochloric acid solution. The dispersed solution shook at a rate of 100 times/min at $37 \pm 5 \stackrel{\circ}{\circ}$. Two milliliters of dissolution samples were collected at $0.083 \mathrm{~h}, 0.25$ h, 1 h, 2 h, 5 h, 12 h, 48 h, 72 h, 120 h, 360 h, 480 h, and 720 $\mathrm{h}$, respectively. Meanwhile, an equal volume of fresh dissolution media was added and maintained at the same temperature to keep the volume of dissolution media constant and to maintain the sink condition. The withdrawn samples were filtered through a cellulose membrane filter $(0.45 \mu \mathrm{m})$ and then analyzed by UV spectrophotometer $\left(\lambda_{\max }=280.0 \mathrm{~nm}\right)$. The experiment was repeated three times.

\section{Results and Discussion}

\section{Process Optimization}

Analysis of the single factor experiment: Five factors, the transmembrane pressure, mild circulation speed, HLB value, PLA concentration and oil-water volume ratio, were investigated in single factor experiments to determine the factors and levels in the orthogonal experiment. The effects of different parameters on these microsphere characteristics are shown in Fig. 4.

\section{Influence of transmembrane pressure on the characteristics of PLA particles}

As transmembrane pressure increased from $0.02 \mathrm{MPa}$ to 0.14 $\mathrm{MPa}$, the average size of the PLA microspheres increased from
$3.95 \mu \mathrm{m}$ to $13.97 \mu \mathrm{m}$ (Fig. 4-A), while the uniformity decreased. SEM photographs of the PLA microspheres with different transmembrane pressure are shown in Fig. 5. Some studies reported that an increase of transmembrane pressure obtained a small size and a narrow size distribution [27-28]. The higher pressure likely caused stronger collisions between the coarse emulsions and the pore walls of the SPG membrane, resulting in stronger droplet breakage.

Previous studies reported that an increase in feed pressure resulted in a gradual increase in the diameter of the emulsion droplets. Higher pressures led to jets of oil and droplet growth and coalescence at the membrane surface due to the decreased interfacial tension [29]. Indeed, the pressure applied to the dispersed phase controlled the flow rate through the SPG membrane pores and the detachment of the droplets. However, when the transmembrane pressure was too low to provide sufficient power to break the large droplets into smaller droplets, large droplets and poor uniformity occurred.

\section{Influence of circulation speed on the characteristics of PLA microspheres}

An increase of circulation speed from $500 \mathrm{rpm}$ to $2000 \mathrm{rpm}$ improved the size distribution, and the average size decreased from $3.96 \mu \mathrm{m}$ to $2.39 \mu \mathrm{m}$ (Fig. 4-B), similar to previous reports [30-31]. SEM photographs of the PLA microspheres with different circulation speeds of the continuous phase are shown in Fig. 6. Compared with circulation speeds of 1500 rpm or 2000 $\mathrm{rpm}$, the surface was rounder and smoother without adhesions at circulation speeds of $500 \mathrm{rpm}$ and $1000 \mathrm{rpm}$.

The faster the circulation speed likely allowed less time for the surfactants to work on the surface of the droplets. Thus, the droplets tended to gathered together. In contrast, a low circulation speed offered enough time for the surfactants and stabilizers to decrease the surface tension, which prevented aggregation of the droplets. Similar results were also observed in previous studies [31-32]. In conclusion, the speed should be high enough to provide the required tangential shear on the membrane surface, but not too excessive to induce further droplet break-up.

\section{Influence of the HLB value on the characteristics of PLA microspheres}

When the HLB value increased from 9.8 to 40 , a small average diameter between $13.88 \mu \mathrm{m}$ and $4.15 \mu \mathrm{m}$ and a narrow size distribution were obtained (Fig. 4-C). This observation suggests that when the HLB value is low, the droplets gradually grow larger to reduce the large surface tension; in contrast, when the HLB value was large, the resulting interfacial tension of the droplets was small enough to make the droplets stable and difficult to coalesce [33]. Based on prior reports, less than $50 \mathrm{mg}$ of the stabilizer is not sufficient to prevent coalescence during nanocapsule formation. In addition, adding more surfactant leads to more stable nanocapsules with less coalescence and lower mean size, but too much surfactant causes the mean diameter of the nanocapsules to increase again [32].

\section{Influence of the PLA concentration on the characteris- tics of PLA microspheres}

Little change in the mean size and span value was observed 

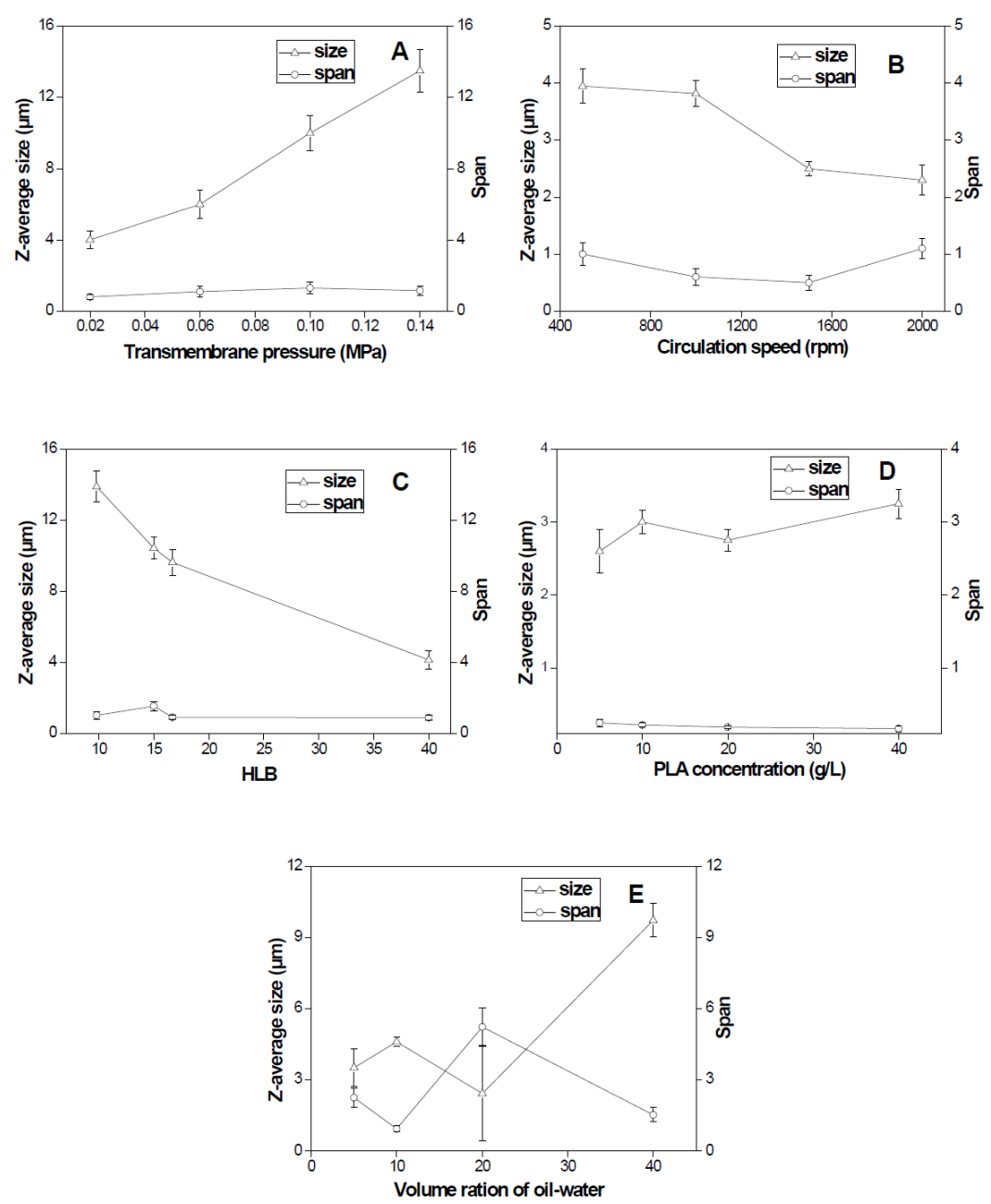

Figure 4: Effect of different parameters on microspheres characteristics: (A) transmembrane pressure, (B) circulation speed, (C) HLB value, (D) PLA concentration, and (E) Volume ratio of oil-water.

3
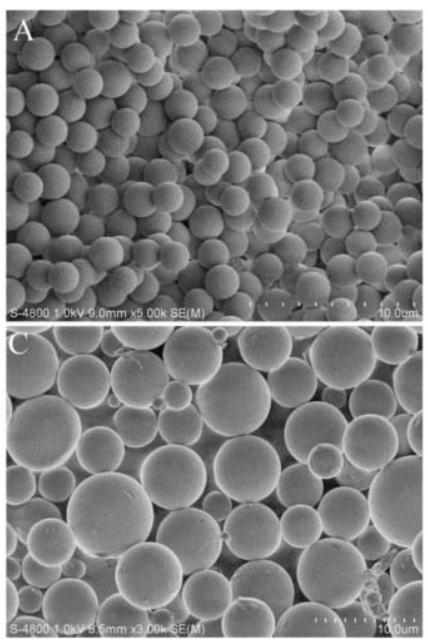

Figure 5: SEM photographs of the PLA microspheres with different

transmembrane pressure: (A) $0.02 \mathrm{MPa},(\mathrm{B}) 0.06 \mathrm{MPa},(\mathrm{C}) 0.10 \mathrm{MPa}$, and (D) $0.14 \mathrm{MPa}$.
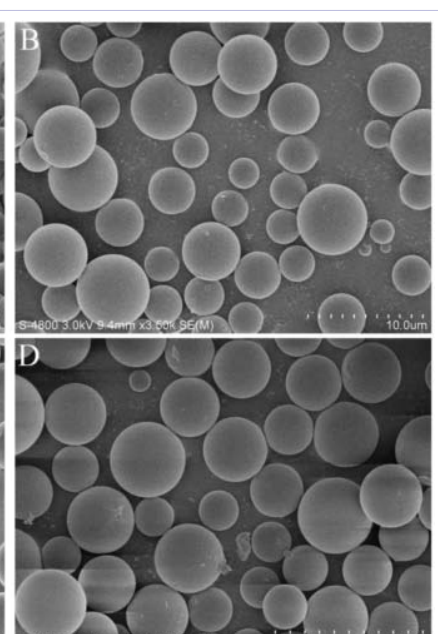

Citation: Lu J, Yin Al, Zhang M, Xue-e Yu, Guo Lw, et al. (2017) Optimization and Evaluation of Monodispersed Tetrandrine-

Loaded PLA Microspheres Prepared With A SPG Membrane Emulsification Technique. SOJ Pharm Pharm Sci, 4(3), 1-11.

DOI: http://dx.doi.org/10.15226/2374-6866/4/3/00148

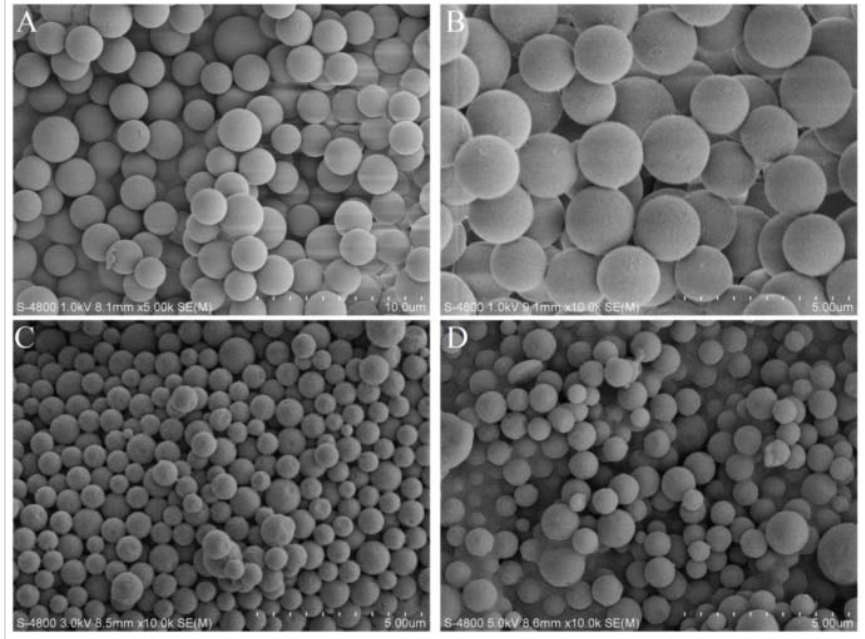

Figure 6: SEM photographs of the PLA microspheres with different circulation speed of the continuous phase: (A) $500 \mathrm{rpm}$, (B) $1000 \mathrm{rpm}$, (C) $1500 \mathrm{rpm}$, and (D) $2000 \mathrm{rpm}$. 
when the PLA concentration was increased from $5 \mathrm{~g} / \mathrm{L}$ to $40 \mathrm{~g} / \mathrm{L}$ (Fig. 4-D). An increase in the PLA concentration may contribute to larger sized droplets that cannot be easily broken due to the increased viscosity at the same pressure. The higher viscosity of the dispersed phase results in larger emulsion droplets [34]. SEM photographs of the PLA microspheres with different PLA concentration are shown in Fig. 7. Interestingly, for a given main condition of the system (especially the pore size of the membrane and the circulation speed), the changed PLA concentration made no significant difference in the size and size distribution of PLA microspheres. The surface of the PLA microspheres at a PLA concentration of $20 \mathrm{~g} / \mathrm{L}$ was more spherical without any fragments just at the PLA concentration of $5 \mathrm{~g} / \mathrm{L}$ and free of the folds observed at PLA concentrations of $10 \mathrm{~g} / \mathrm{L}$ and $40 \mathrm{~g} / \mathrm{L}$.

\section{Influence of the oil-water volume ratio on PLA microspheres}

The oil-water volume ratio played a role in the size and size distribution of the PLA microspheres; a decrease of the volume ratio from 1:5 to 1:40 revealed larger sizes, from $3.52 \mu \mathrm{m}$ to 9.73 $\mu \mathrm{m}$, and a broader size distribution (Fig. 4-E). SEM photographs of the PLA microspheres with different oil-water volume ratios are shown in Fig. 8. Similar results have been reported, in which the oil droplet became viscous, weakening the extent of droplet disruption of the coarse emulsions [34]. When the ratio is low, dichloromethane is easy to evaporate in the continuous phase of low volume and the process of droplet solidification is short, which is beneficial to prevent the droplets from coalescing. In contrast, the larger the volume, the longer the time for the dichloromethane to evaporate, thus the higher the probability that the droplets will gather, leading to larger size and a broader size distribution.

\section{Analysis of the orthogonal experiment}

Microspheres were characterized in terms of their morphology $\left(\mathrm{Y}_{1}\right.$, total score $=1$, the morphology of desirable microspheres are smooth and round), span value $\left(\mathrm{Y}_{2}\right)$, loading efficiency $\left(\mathrm{Y}_{3}\right.$, total score $\left.=1\right)$ and encapsulation efficiency $\left(\mathrm{Y}_{4}\right.$, total score =1), where $Y=Y_{1}-Y_{2}+Y_{3}+Y_{4}$. Desirable microspheres were evaluated with a high $Y$ value. As seen from Tables 3 and 4 , the degree of influence of the four factors was: D (oil-water volume ratio) $>\mathrm{A}$ (PLA concentration) $>\mathrm{B}$ (concentration ratio of tetrandrine-PLA) $>$ C (SDS). Variance analysis indicated that the oil-water volume ratio had the strongest impact, followed by the PLA concentration; the tetrandrine-PLA concentration ratio showed the weakest impact, while the SDS concentration had no significant impact on the characteristics of tetrandrine-loaded PLA microspheres. Thus, the optimized condition included an oil-water volume ratio of 1:10, a PLA concentration of 60 $\mathrm{g} / \mathrm{L}$, a tetrandrine-PLA concentration ratio of $1: 6$ and an SDS concentration of $4 \mathrm{~g} / \mathrm{L}$.

The influence of the PLA concentration and oil-water volume ratio were analyzed above. SDS was used as an emulsifier to prevent the droplets from coalescing. The gradient of SDS concentration set in the experiment may not have been high enough to make a difference. SDS concentrations have not been reported to correlate with the viscosity of the formulation and the interfacial tension between the two phases [35-36]. An increase in the surfactant amount produced an increase in the
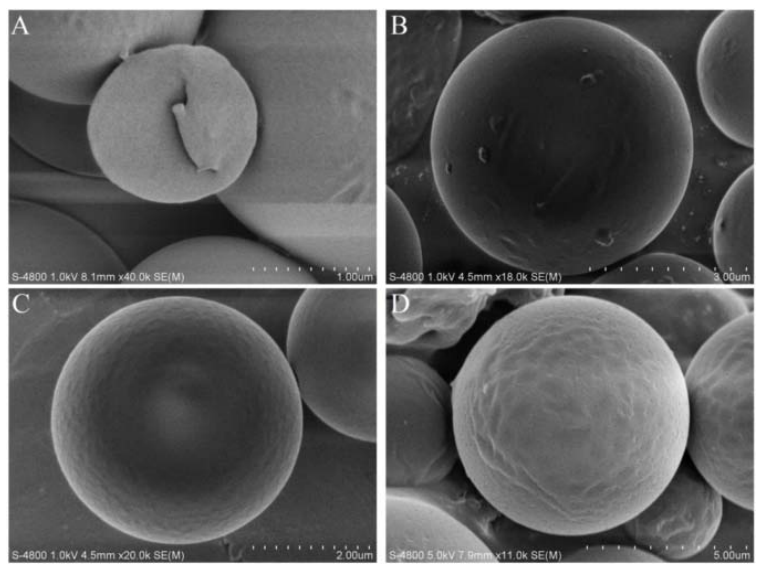

Figure 7: SEM photographs of the PLA microspheres with different PLA concentration: (A) $5 \mathrm{~g} / \mathrm{L},(B) 10 \mathrm{~g} / \mathrm{L},(C) 20 \mathrm{~g} / \mathrm{L}$, and (D) $40 \mathrm{~g} / \mathrm{L}$.
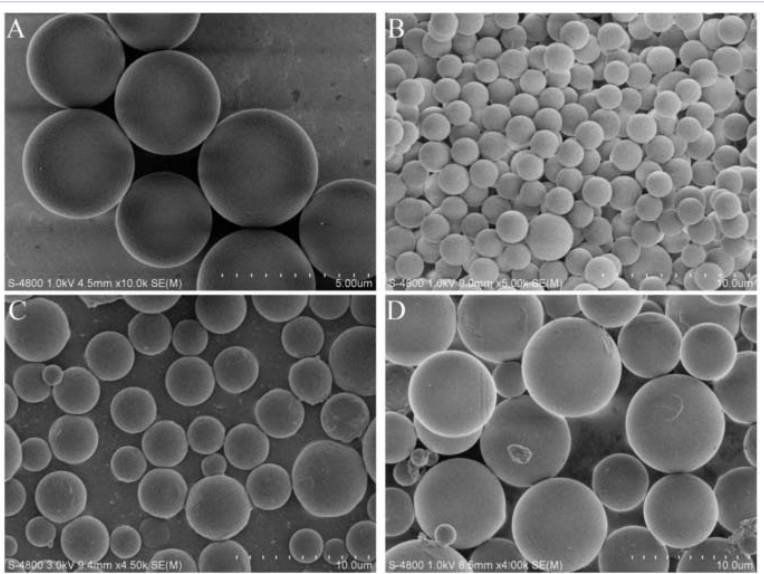

Figure 8: SEM photographs of the PLA microspheres with different volume ratio of oil-water: (A) 1:5, (B) 1:10, (C) 1:20, and (D) 1:40.

\begin{tabular}{|c|c|c|c|c|c|}
\hline \multirow{2}{*}{$\begin{array}{l}\text { Table 3: Intuitive analysis. } \\
\text { Experiment } \\
\text { Number }\end{array}$} & A & B & C & D & \\
\hline 1 & 1 & 1 & 1 & 1 & 0.631 \\
\hline 2 & 1 & 2 & 2 & 2 & 0.318 \\
\hline 3 & 1 & 3 & 3 & 3 & 0.039 \\
\hline 4 & 2 & 1 & 2 & 3 & 0.008 \\
\hline 5 & 2 & 2 & 3 & 1 & 0.401 \\
\hline 6 & 2 & 3 & 1 & 2 & 0.619 \\
\hline 7 & 3 & 1 & 3 & 2 & 0.916 \\
\hline 8 & 3 & 2 & 1 & 3 & 0.254 \\
\hline 9 & 3 & 3 & 2 & 1 & 1.055 \\
\hline $\mathrm{K}_{1}$ & 3.293 & 5.183 & 5.013 & 6.957 & \\
\hline $\mathrm{K}_{2}$ & 3.427 & 3.243 & 4.603 & 6.177 & \\
\hline $\mathrm{K}_{3}$ & 7.417 & 5.710 & 4.520 & 1.003 & \\
\hline $\mathrm{R}$ & 4.124 & 2.467 & 0.493 & 5.954 & \\
\hline & & & & & \\
\hline
\end{tabular}


nanocapsules' mean size [32]. A high tetrandrine concentration provided a great opportunity for the PLA to encapsulate molecular tetrandrine. As long as the tetrandrine concentration was kept within the encapsulating capacity of PLA and the stabilized capacity of the surfactant and stabilizer in the continuous phase, a higher concentration resulted in a high loading efficiency and high encapsulation efficiency. Meanwhile, the viscosity of the formulation was also affected by the tetrandrine concentration, thus preventing dichloromethane from evaporating through $\mathrm{O} / \mathrm{W}$ emulsions, which resulted in an increase in microspheres size.

\section{The reproducibility of tetrandrine-loaded PLA microspheres}

SEM photographs of tetrandrine-loaded PLA microspheres prepared at the optimized conditions are shown in Fig. 9. The uniformly sized tetrandrine-loaded microspheres were highly reproducible, with an average diameter of $3.16 \mu \mathrm{m}$ and a span value of 0.67 (Table 5), which corresponds with the particle size requirement for pulmonary inhalation and demonstrates that a suppressed and precise release could be obtained with a narrow size distribution [32]. Tetrandrine showed good solubility in the oil phase, and PLA could protect the internal tetrandrine from leaking to obtain a high encapsulation efficiency of $81.0 \%$.

\section{The preliminary stability study of tetrandrine-loaded PLA microspheres}

The freeze-dried tetrandrine-loaded PLA microspheres were kept at $-20{ }^{\circ} \mathrm{C}, 4{ }^{\circ} \mathrm{C}$ and $37{ }^{\circ} \mathrm{C}$ for 1,2 and 3 months to evaluate their stability [37]. The preliminary stability results are shown in Table 6. No significant changes were found in the encapsulation efficiency and microscopic appearance of these microspheres during the storage period of $-20{ }^{\circ} \mathrm{C}$ or $4{ }^{\circ} \mathrm{C}$, indicating that it was stable for at least 3 months at these conditions. However, the microspheres kept at $37{ }^{\circ} \mathrm{C}$ showed conglutination with each other after only 1 month. We speculated that when kept at 37 oC, PLA may decompose at a different rate. In addition, a small amount of the residual organic solvent evaporated outside the microspheres, allowing the tetrandrine to coalesce at the surface of these microspheres. Therefore, in our study, we chose to store the microspheres at $-20{ }^{\circ} \mathrm{C}$ or $4 \stackrel{\circ}{\circ} \mathrm{C}$ for at least 3 months.

\section{FTIR spectroscopy}

The FTIR spectra of native tetrandrine, a physical mixture of tetrandrine and PLA, tetrandrine-free PLA microspheres and tetrandrine-loaded PLA microspheres are shown in Fig. 10. As

Table 4: Variance analysis.

\begin{tabular}{|c|c|c|c|c|c|}
\hline Factors & $\begin{array}{c}\text { Sum of } \\
\text { squares }\end{array}$ & $\begin{array}{c}\text { Degree of } \\
\text { freedom }\end{array}$ & F-value & $\mathbf{F}_{\mathbf{0}}$ & Significant \\
\hline A & 32.94 & 2 & 78.804 & 19.000 & $*$ \\
\hline B & 10.125 & 2 & 34.222 & 19.000 & $*$ \\
\hline C & 0.418 & 2 & 1 & 19.000 & - \\
\hline D & 62.814 & 2 & 150.273 & 19.000 & $*$ \\
\hline R & 0.42 & 2 & & & \\
\hline
\end{tabular}

Note: * for significant influence, - for no significant influence.

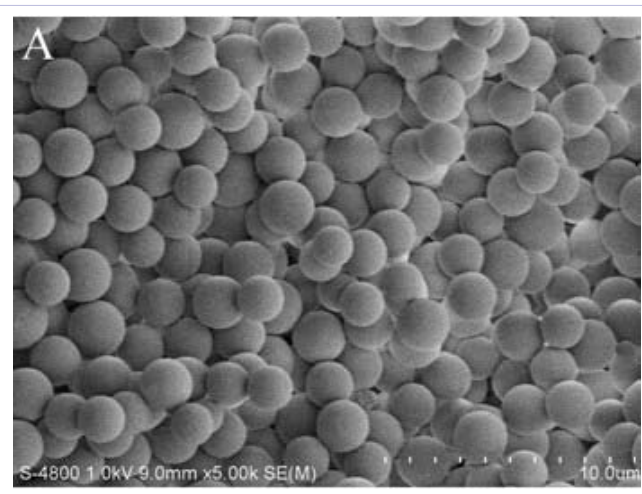

Figure 9: SEM photographs of tetrandrine-loaded PLA microspheres.

Table 5: Characteristics of tetrandrine-loaded PLA microspheres at the optimized condition

\begin{tabular}{|c|c|c|c|c|}
\hline $\begin{array}{c}\text { Experiment } \\
\text { number }\end{array}$ & $\begin{array}{c}\text { Loading } \\
\text { efficiency }\end{array}$ & $\begin{array}{c}\text { Encapsulation } \\
\text { efficiency }\end{array}$ & $\begin{array}{c}\text { Average size } \\
(\boldsymbol{\mu m})\end{array}$ & Span value \\
\hline 1 & 0.132 & 0.820 & 3.04 & 0.63 \\
\hline 2 & 0.125 & 0.830 & 3.32 & 0.71 \\
\hline 3 & 0.108 & 0.780 & 3.13 & 0.67 \\
\hline Mean & 0.122 & 0.810 & 3.16 & 0.67 \\
\hline SD & 0.012 & 0.026 & 0.143 & 0.040 \\
\hline
\end{tabular}

Table 6: The preliminary stability experiment result of tetrandrineloaded PLA microspheres

\begin{tabular}{|c|c|c|c|}
\hline $\begin{array}{c}\text { Temperature } \\
\mathbf{( \mathbf { o }} \mathbf{C})\end{array}$ & Month & $\begin{array}{c}\text { Encapsulation } \\
\text { efficiency (\%)* }\end{array}$ & $\begin{array}{c}\text { Microscopic } \\
\text { appearance }\end{array}$ \\
\hline-20 & 1 & $79.61 \pm 1.780$ & + \\
\hline-20 & 2 & $82.34 \pm 1.821$ & + \\
\hline-20 & 3 & $80.41 \pm 1.032$ & + \\
\hline 4 & 1 & $79.54 \pm 2.644$ & + \\
\hline 4 & 2 & $80.43 \pm 2.183$ & + \\
\hline 4 & 3 & $82.19 \pm 1.955$ & + \\
\hline 37 & 1 & $75.63 \pm 2.582$ & 0 \\
\hline 37 & 2 & $64.38 \pm 1.324$ & - \\
\hline 37 & 3 & $48.92 \pm 0.591$ & - \\
\hline
\end{tabular}

Note: *Each value represents the mean \pm S.D. $(n=3)$. Tet-loaded PLA microspheres were graded with the following three symbols: + = very good, 0 = good, - = bad.

shown in Fig. 10-A, the spectral analysis of native tetrandrine showed main peaks at $1450-1600 \mathrm{~cm}^{-1}$ and $1020-1310 \mathrm{~cm}^{-1}$, corresponding to the vibration absorption peak of the benzene skeleton and the stretching vibration of the ether bond; $1750 \mathrm{~cm}^{-1}$ was attributed to the characteristic $-\mathrm{C}=\mathrm{O}$ stretching vibrations of PLA in tetrandrine-free PLA microspheres. The characteristic absorption peaks of tetrandrine in the physical mixture were weaker in spectrum compared to that of native tetrandrine. The spectra of tetrandrine-loaded PLA microspheres were similar to those of tetrandrine-free PLA microspheres. Although the vibration spectral peaks of the drug in the spectrum of tetrandrine- 

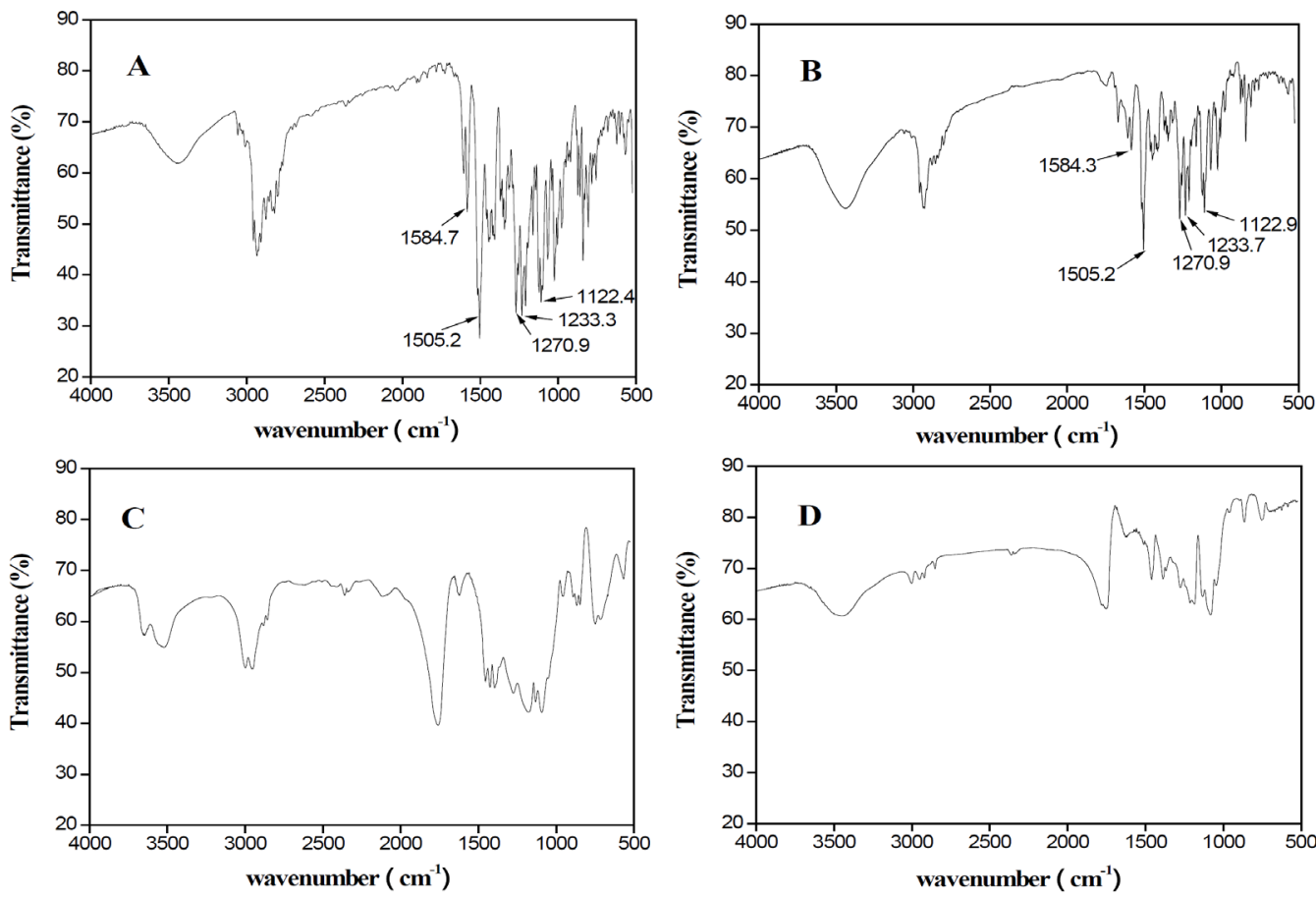

Figure 10: SEM photographs of tetrandrine-loaded PLA microspheres.

loaded PLA microspheres were not observed, a strong infrared absorption peak at 1600 to $1700 \mathrm{~cm}^{-1}$ was observed, which may cover the characteristic absorption peaks. More characterization methods will be needed to evaluate tetrandrine - loaded PLA microspheres.

\section{Differential scanning calorimetry}

DSC is a thermal analytical technique that provides information about the physical properties, such as the crystalline or amorphous nature of the samples [38]. The basic principle behind this technique is that when the sample undergoes a physical transformation (such as melting, crystal transformation, desolvation), the system will be changed, changing its enthalpy, resulting in thermal effects. DSC measures the heat flow into or from the sample when it is heated or cooled and then provides qualitative and quantitative information about physicochemical changes [39]. There is no detectable endotherm if the drug is present in a molecular dispersion or solid solution state in polymeric microspheres [40].

DSC thermograms of native tetrandrine, a physical mixture of tetrandrine and PLA, tetrandrine-free PLA microspheres and tetrandrine-loaded PLA microspheres are shown in Fig. 11. Native tetrandrine shows a sharp endothermic peak at 225.5 ${ }^{\circ} \mathrm{C}$, which is closer to its melting point $\left(217-218{ }^{\circ} \mathrm{C}\right)$, indicating a melting effect of native tetrandrine and proving its crystalline nature of tetrandring ${ }^{41}$. In addition, native tetrandrine shows an exothermic peak at $344.7^{\circ} \mathrm{C}$. Tetrandrine-free PLA microspheres show an endothermic peak indicative of the polymer transition temperature at $56.6{ }^{\circ} \mathrm{C}$, and an exothermic peak at $292.6{ }^{\circ} \mathrm{C}$.
Comparing the thermal analysis curves of native tetrandrine and the physical mixture of tetrandrine and PLA, the endothermic peak and exothermic peak tetrandrine in physical mixture were transferred to $218.1{ }^{\circ} \mathrm{C}$ and $307.5{ }^{\circ} \mathrm{C}$ respectively. The endothermic peak of PLA in the physical mixture was transferred to $277.7^{\circ} \mathrm{C}$. The intensity of these three peaks was weakened. Tetrandrine-loaded PLA microspheres only show an endothermic peak indicative of the polymer transition temperature at $66.6{ }^{\circ} \mathrm{C}$ and an endothermic peak at $362.1^{\circ} \mathrm{C}$. No characteristic peaks of native tetrandrine were observed, indicating that tetrandrine would be either molecularly dispersed in the polymer or distributed in an amorphous form.

\section{Powder X-ray diffraction study}

The powder X-ray diffraction (XRD) spectra of native tetrandrine, a physical mixture of tetrandrine and PLA, tetrandrine-free PLA microspheres and tetrandrine-loaded PLA microspheres are shown in Fig. 12. Several distinct picks in the XRD of native tetrandrine at diffraction angles of $7^{\circ}-28^{\circ}$, indicate that native tetrandrine was present in a crystalline form, whereas that of PLA shows the amorphous nature. The angles about tetrandrine could be observed in the XRD spectrum of the physical mixture. However, these angles disappeared in the XRD spectrum of the tetrandrine-loaded PLA microspheres, indicating that tetrandrine distributed in the polymer in an amorphous form [42].

\section{In vitro tetrandrine release profiles}

The in vitro release profiles from the tetrandrine-loaded PLA microspheres were characterized by an initial fast release. 
Drug release from biodegradable polymeric particles occurred through a combination of several mechanisms: surface-bound drug was desorbed from the microspheres' surface, encapsulated drug was diffused through the polymer matrix and the polymer particles degraded gradually. Doan et al., 2011 also reported that higher polymer concentrations resulted in a higher burst release [19]. In this study, the burst release was approximately 35\% for the tetrandrine-loaded microspheres (loading efficiency of microspheres is $12.2 \%$.) within $5 \mathrm{~h}$. The in vitro release profiles of tetrandrine-loaded PLA microspheres are shown in Fig. 13. In general, drug released in the initial stage of the release is located at the surface of the microspheres. Thus, a low initial burst release suggests a lower drug density at the surface of the microspheres [42], which suggests that tetrandrine was distributed homogenously among the PLA microspheres. Moreover, approximately $5 \%$ of tetrandrine was released in the 30 days after being maintained in the hydrochloric acid solution. It was confirmed that narrow size distributed tetrandrine-loaded PLA microspheres prepared by this novel method showed suppressed and sustained release.

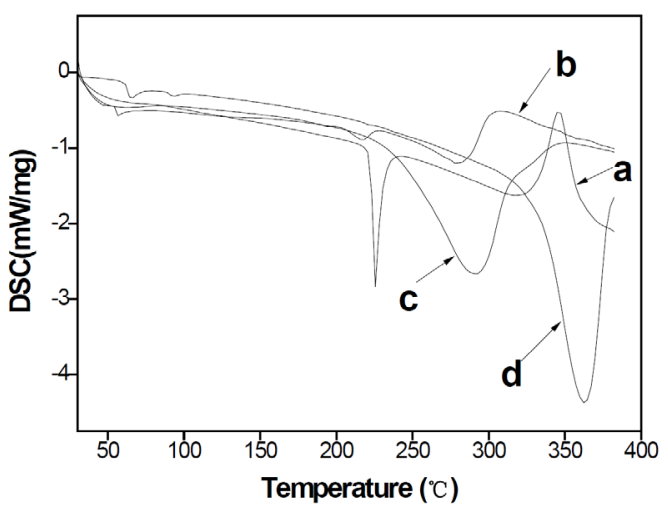

Figure 11: Differential scanning calorimetry (DSC) thermogram of (a) native tetrandrine, (b) physical mixture of tetrandrine and PLA, (c) tetrandrine-free PLA microspheres, (d) tetrandrine-loaded PLA microspheres.

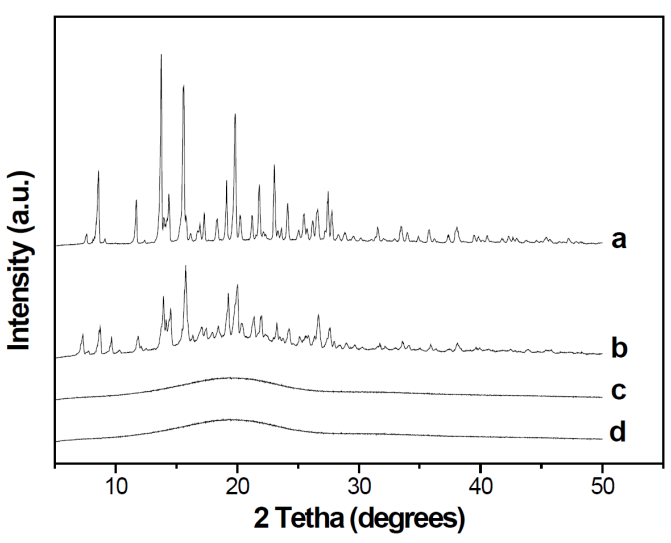

Figure 12: The XRD spectra of (a) native tetrandrine, (b) physical mixture of tetrandrine and PLA, (c) tetrandrine-free PLA microspheres, (d) tetrandrine-loaded PLA microspheres.

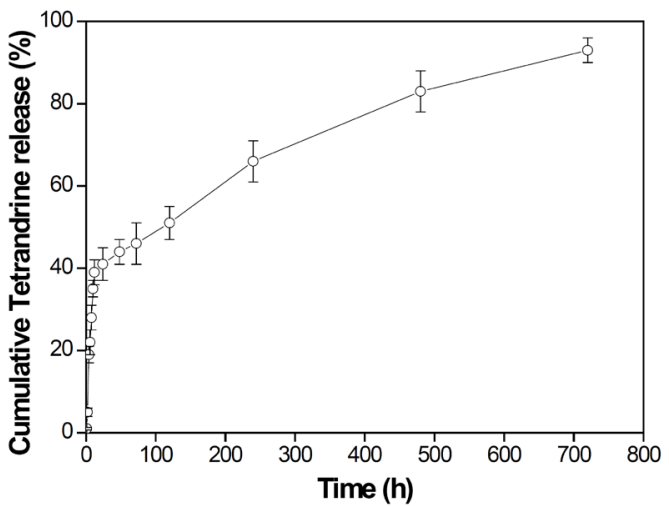

Figure 13: In vitro release profiles of tetrandrine-loaded PLA microspheres.

\section{Conclusions}

For the first time, we have prepared uniform-sized microspheres containing tetrandrine, the main active ingredient of Fangji, by SPG membrane emulsification. Researchers have applied this novel method in other active components of traditional Chinese medicine, such as paclitaxel [27], berberine [43] and silybin [44], with drug encapsulation efficiencies of $63.2 \%, 32.57 \%, 56.16 \%$, respectively. Tetradrine-tashionone $\mathrm{II}_{\mathrm{A}^{-}}$ PLGA composite microspheres prepared by the SPG membrane emulsification method was reported [45]. However, the encapsulation efficiency of tetrandrine-loaded PLA microspheres prepared at the optimized parameters was elevated to $81.0 \%$, which would achieve a significant level and meet the actual demands of a drug delivery system. FTIR, DSC and X-ray diffraction show that tetrandrine would be either molecularly dispersed in the polymer or distributed in an amorphous form. Drug loading is closely related to the presence of drugs in the form of microspheres. It also showed differences associated with release in vitro [46]. Furthermore, the drug-loaded microspheres' stability and drug release (in vitro) were also investigated to complete the whole evaluation system. The uniformly sized tetrandrine-loaded PLA microspheres of $3.16 \mu \mathrm{m}$ prepared by SPG membrane provide a possible route of lung targeting after pulmonary inhalation.

\section{Acknowledgment}

This work was supported by A Project Funded by Innovation Research Team of Nanjing University of Chinese Medicine (No. 012062003018-6; No. 012062003018-9), the Natural Scientific Fund of China (No.81303230) and A Project Funded by the Priority Academic Program Development of Jiangsu Higher Education Institutions (PAPD).

\section{Declaration of interest}

No completing financial interests exist.

\section{References}

1. Pharmacopoeia Commission of Republic. Pharmacopoeia of the People's Republic of China (Part I). Chemical Industry Press: Beijing. China. 2010. 
2. Tang Y, Sun A, Liu R, Zhang Y. Simultaneous determination of fangchinoline and tetrandrine in Stephania tetrandra S. Moore by using1-alkyl-3-methylimidazolium-based ionic liquids as the RPHPLC mobile phase additives. Analytica chimica acta. 2013;767:148154.

3. Cai X, Wang S, Chen B. Research advances on the pharmacological effect of tetrandrine. Chinese Journal of Natural Medicines. 2011;9(6):473480 .

4. Wei X, Pei G, Lin Y, Ding Y, Tang X, Zhang J. Research on the growth of A549 cells inhibited by tetrandrine. Chinese Journal of Modern Drug Application. 2010;4:105-106

5. Sun X, Cao Y, Cheng H, Yu D, Ge X, Li Y, et al. The clinical research on tetrandrine for radiosensitization on non-small cell lung cancer. Chinese Journal of Clinical Oncology. 2007;12:753-756.

6. Zhu L, Li R, Yu L, Qian X, Liu B. Novel nanoparticle of tetrandrine preparation and properties. Modern Oncology. 2009;17:798-801.

7. Xu X, Gan Y, Xu G, Chen T, Zhou H, Tang J, et al. Tetrandrine citrate eliminates imatinib-resistant chronic myeloid leukemia cells in vitro and in vivo by inhibiting Bcr-Abl/ $\beta$-catenin axis. Journal of Zhejiang University Science B. 2012;13(11): 867-874. doi: 10.1631/jzus. B1200021.

8. Cheng G, Luo J. Study on distribution in mouse of tetrandrine polylactic acid microsphere in vivo. China Pharmacy. 2005;16(6):418-420

9. Liu R, Ma G, Meng F, Su Z. Preparation of uniform-sized PLA microcapsules by combining shirasu porous glass membrane emulsification technique and multiple emulsion-solvent evaporation method. Journal of Controlled Release. 2005;103(1):31-43.

10. Ito F, Honnarmi H, Kawakami H, Kanamura K, Makino K. Preparation and properties of PLGA microspheres containing hydrophilic drugs by the SPG(shirasu porous glass) membrane emulsification technique. Colloids and Surfaces B: Biointerfaces. 2008;67(1):20-25. doi: 10.1016/j.colsurfb.2008.07.008.

11. Surh J, Jeong YG, Vladisavljević GT. On the preparation of lecithinstabilized oil-in-water emulsions by multi-stage premix membrane emulsification SPG membranes. Journal of food engineering. 2008;89(2):164-170.

12. Pathak M. Numerical simulation of membrane emulsification: Effect of flow properties in the transition from dripping to jetting. Journal of membrane science. 2011;382(1-2):166-176.

13. Chu T, Zhang Q, Li H, Ma W, Zhang N, Jin H, et al. Development of intravenous lipid emulsion of tanshinone IIA and evaluation of its antihepatoma activity in vitro. International journal of pharmaceutics. 2012;424(1-2):76-88. doi: 10.1016/j.ijpharm.2011.12.049.

14. Joseph S, Bunjes H. Preparation of nanoemulsions and solid lipid nanoparticles by premix membrane emulsification. Journal of pharmaceutical sciences. 2012;101(7):2479-2489. doi: 10.1002/ jps.23163.

15. Duret C, Wauthoz N, Sebti T, Vanderbist F, Amighi K. Solid dispersions of itraconazole for inhalation with enhanced dissolution, solubility and dispersion properties. International journal of pharmaceutics. 2012;428(1-2):103-113. doi: 10.1016/j.ijpharm.2012.03.002

16. Healy AM, Amaro MI, Paluch KJ, Tajber L. Dry powders for oral inhalation free of lactose carrier particles. Advanced drug delivery reviews. 2014;75: 32-52. DOI: 10.1016/j.addr.2014.04.005

17. Nakashima T, Shimizu M, Kukizaki M. Membrane emulsification by microporous glass. Key Engineering Materials. Trans Tech Publications. 1992;61:513-516.
18. Koocheki A, Kadkhodaee R, Mortazavi SA, Shahidi F, Taherian AR Influence of Alyssum homolocarpum seed gum on the stability and flow properties of $0 / \mathrm{W}$ emulsion prepared by high intensity ultrasound. Food Hydrocolloids. 2009; 23(8): 2416-2424. DOI: 10.1016/j.foodhyd.2009.06.021

19. Doan TVP, CouetW, Olivier JC. Formulation and in vitro characterization of inhalable rifampicin-loaded PLGA microspheres for sustained lung delivery. International journal of pharmaceutics. 2011; 414(1):112117.

20. Yuyama H, Watanabe $\mathrm{T}$, Ma GH, Nagai M, Omi S. Preparation and analysis of uniform emulsion droplets using SPG membrane emulsification technique. Colloids and Surfaces A: Physicochemical and Engineering Aspects. 2000;168(2):159-174. DOI: 10.1016/ S0927-7757(00)00452-0

21. Kukizaki M, Goto M. Preparation and evaluation of uniformly sized solid lipid microcapsules using membrane emulsification. Colloids and Surfaces A: Physicochemical and Engineering Aspects. 2007;293(1):87-94. DOI: 10.1016/j.colsurfa.2006.07.011

22. Akamatsu K, Chen W, Suzuki Y, Ito T, Nakao A, Sugawara T, Kikuchi R, Nakao S. Preparation of monodisperse chitosan microcapsules with hollow structures using SPG membrane emulsification technique. Langmuir. 2010; 26(18):14854-14860. DOI: 10.1021/la101967u

23. Akamatsu K, Ikeuchi Y, Nakao A, Nakao S. Size-controlled and monodisperse enzyme-encapsulated chitosan microspheres developed by the SPG membrane emulsification Technique. Journal of colloid and interface science. 2012;371(1): 46-51. doi: 10.1016/j. jcis.2011.12.078.

24. Wang Y. Monodisperse microspheres as drug release system prepared by Shirasu Porous glass (SPG). XiaMen: Xiamen University. 2008.

25. Vladisavljević G T, Schubert H. Influence of process parameters on droplet size distribution in SPG membrane emulsification and stability of prepared emulsion droplets. Journal of membrane science. 2003;225(1): 15-23. doi.org/10.1016/S0376-7388(03)00212-6.

26. Huang S, Liu R, Ma G, Tan T. Preparation of uniform-sized PLA/ PLGA microcapsules containing lysozyme by combining porous glass membrane emulsification and multiple emulsion-solvent evaporation. Chinese Journal of Process Engineering. 2006; 6(4): 603-607.

27.Zeng Y, Wang L, Ma G. Preparation of microspheres of paclitaxelloaded PLA, PLGA and PELA by premix membrane emulsification. The Chinese Journal of Process Engineering. 2010;3:26.

28. Kou X, Li Q, Lei J, Geng L, Deng H, Zhang G, et al. Preparation of molecularly imprinted nanospheres by premix membrane emulsification technique. Journal of membrane science. 2012;417418:87-95.

29. Choi YK, Poudel BK, Marasini N, Yang KY, Kim JM, Kim JO, et al. Enhanced solubility and oral bioavailability of itraconazole by combining membrane emulsification and spray drying technique. International journal of pharmaceutics. 2012;434(1):264-271. doi: 10.1016/j.ijpharm.2012.05.039.

30. Gasparini G, Kosvintsev SR, Stillwell MT, Holdich RG. Preparation and characterization of PLGA particles for subcutaneous controlled drug release by membrane emulsification. Colloids and surfaces B: biointerfaces. 2008;61(2):199-207.

31. Laouini A, Fessi H, Charcosset C. Membrane emulsification: A promising alternative for vitamin $\mathrm{E}$ encapsulation within nanoemulsion. Journal of membrane science. 2012;423-424:85-96. 
32. Khayata N, Abdelwahed W, Chehna MF, Charcosset C, Fessi H. Preparation of vitamin $\mathrm{E}$ loaded nanocapsules by the nanoprecipitation method: From laboratory scale to large scale using a membrane contactor. International journal of pharmaceutics. 2012;423(2):419427. doi: 10.1016/j.ijpharm.2011.12.016.

33. Vladisavljević GT, McClements DJ. Modification of interfacial characteristics of monodisperse droplets produced using membrane emulsification by surfactant displacement and/or polyelectrolyte electrostatic deposition. Colloids and Surfaces A: Physicochemical and Engineering Aspects. 2010;364(1): 123-131.

34. Wei Q, Wei W, Lai B, Wang LY, Wang YX, Su ZG, et al. Uniform-sized PLA nanoparticles preparation by premix membrane emulsion. International journal of pharmaceutics. 2008;359(1-2):294-297.

35. Meyer RF, Rogers WB, McClendon MT, Crocker JC. Producing monodisperse drug-loaded polymer microspheres via cross-flow membrane emulsification: The effects of polymers and surfactants. Langmuir. 2010;26(18):14479-14487. doi: 10.1021/la1023136.

36. Joseph S, Bunjes $H$. Influence of membrane structure on the preparation of colloidal lipid dispersions by premix membrane emulsification. International journal of pharmaceutics. 2013;446(12):59-62.

37. Bouchemal K, Briançon S, Perrier E, Fessi H, Bonnet I, Zydowicz $\mathrm{N}$. Synthesis and characterization of polyurethane and poly (etherurethane) nanocapsules using a new technique of interfacial polycondensation combined to spontaneous emulsification. International Journal of Pharmaceutics. 2004;269(1):89-100.

38. Mo L, Hou L, Guo D, Xiao X, Mao P, Yang X. Preparation and characterization of teniposide PLGA nanoparticles and their uptake in human glioblastoma U87MG cells. International journal of pharmaceutics. 2012;436(1-2):815-824. doi: 10.1016/j. ijpharm.2012.07.050.
39. Kumar M, Kakkar V, Mishra AK, Chuttani K, Kaur IP. Intranasal delivery of streptomycin sulfate (STRS) loaded solid lipid nanoparticles to brain and blood. International journal of pharmaceutics. 2014;461(1):223233. doi: 10.1016/j.ijpharm.2013.11.038.

40. Dubernet C. Thermoanalysis of microspheres. Thermochimica Acta. 1995;248:259-269.

41. Seju U, Kumar A, Sawant KK. Development and evaluation of olanzapine-loaded PLGA nanoparticles for nose-to-brain delivery: In vitro and in vivo studies. Acta Biomaterialia. 2011;7(12):4169-4176. doi: 10.1016/j.actbio.2011.07.025.

42. Nath SD, Son S, Sadiasa A, Min YK, Lee BT. Preparation and characterization of PLGA microspheres by the electrospraying method for delivering simvastatin for bone regeneration. International journal of pharmaceutics. 2013;443(1-2):87-94.

43. $\mathrm{Li} \mathrm{H} \mathrm{Q}$, Yi T. Preparation and in vitro characterization of berberine hydrochloride-self-emulsifying microsphere by membrane emulsification technology. Acta pharmaceutica Sinica. 2013;48(4):554-559.

44. He Y, Liu B, Li M, Rong K, Cai K. Optimization of preparation technology of silybin PLGA microspheres by premix membrane emulsificationsolvent extraction/evaporation method. Chinese Journal of Experimental Traditional Medical Formulae. 2013;19(1):1-6.

45. Lu J, Zhang M, Zhu HX, Guo LW, Pan LM, Fu TM. Studies on preparation by SPG membrane emulsification method and in vitro characterization of tetradrine-tashionone $\mathrm{II}_{\mathrm{A}}$-PLGA composite microspheres. China journal of Chinese materia medica. 2015;40(6):1091-1096.

46. Chen D, Liu Y, Fan KY, Xie YQ, Yu A, Xia ZH, et al. Relation between drug release and the drug status within curcumin-loaded microsphere. Acta pharmaceutica Sinica. 2016;51(1):140-146. 